G. L. Csordas and H. B. Reiter

Nagoya Math. J.

Vol. 47 (1972), 101-109

\title{
SEPARATING FUNCTION ALGEBRAS
}

\author{
G. L. CSORDAS AND H. B. REITER
}

1. Introduction. Recent results of Hoffman and Singer [7], Weiss [10] and Wilken [11] indicate that the study of separation properties play a central rôle in the theory of function algebras. Our purpose in this paper is to investigate a natural separation property of function algebras.

Let $A$ be a sup-norm algebra of complex-valued continuous functions on a compact Hausdorff space $X$. In the sequel we will call a closed subset $S$ of $X$ an $L_{A^{-}}$set or briefly an $L$-set, if $L(S)=S$, where

$$
L(S)=\bigcap_{f \in A} f^{-1} f(S) .
$$

We will say that an algebra $A$ on $X$ is a separating algebra if every closed subset of $X$ is an $L$-set. Clearly, regular, approximately normal and approximately regular algebras are all examples of separating algebras. (For the terminology used here we refer the reader to Wilken [11].) In fact, any algebra that contains a one-one function is a separating algebra. As we will see in section 4, pervasive, Dirichlet and maximal algebras are also examples of separating algebras. The concept of a separating algebra is quite broad as the example of the disk algebra-the algebra of all continuous functions on the closed disk which are analytic on the open disk $D$-shows. For although, the disk algebra is a separating algebra it is neither maximal nor approximately regular.

On the other hand, in general, it is difficult to determine whether an algebra is a separating algebra. By way of illustration consider the algebra $H^{\infty}$ of all bounded analytic functions on the open disk $D$. Is $H^{\infty}$ considered as an algebra of functions on its maximal ideal space a separating algebra? An affirmative answer to this question together with an elementary argument from the theory of cluster sets would imply the Corona Theorem (see [3]).

2. Definitions. In the sequel we will use the following terminology and notation. $X$ will denote a compact Hausdorff space and $C(X)$ the Received May 7, 1971. 
Banach algebra of all continuous complex-valued functions on $X$ with the usual supremum norm. We will say that $A$ is an algebra on $X$ if $A$ is a closed subalgebra of $C(X)$, contains the constant functions and separates points of $X$. We will say that $A$ is a maximal algebra on $X$ if $A$ is an algebra on $X$ and $A$ is contained in no other proper closed subalgebra of $C(X)$.

We will denote by $M_{A}$ the maximal ideal space of $A$ endowed with the Gelfand topology. As is customary, we will identify each maximal ideal in $M_{A}$ with the complex-valued algebra homomorphism that it determines. If $A$ is an algebra on $X$, then $M_{A}$ is a compact Hausdorff space, and $X$ is homeomorphic to a subset of $M_{A}$. We will denote by $\Gamma_{A}$ the Silov boundary of $A$.

If $S$ is a closed subset of $X$ we will denote by $f \mid S$ the restriction of the function $f$ to $S$ and by $A \mid S$ the algebra of restrictions of functions in $A$. $A_{S}$ will denote the completion of $A \mid S$ in the sense of uniform convergence on $S$.

The essential set of $A$ in $X$ is that unique minimal closed subset $E$ of $X$ such that if $f$ is any continuous function of $X$ and $f|E \in A| E$, then $f \in A$. Finally, we will say that $A$ is an essential algebra on $X$, if the essential set for $A$ is all of $X$. This terminology is due to Bear [1].

3. Properties of $\boldsymbol{L}$-sets. Let $A$ be an algebra on $X$. Evidently, the whole space $X$, each singleton, $\{x\}$, and any finite set, $T=\left\{x_{1}, \cdots, x_{n}\right\}$, are $L$-sets. Next we show that the closed set obtained by adding a finite set of points to an $L$-set is also an $L$-set.

THEOREM 3.1. Let $S$ be an L-set, then

$$
L(S \cup T)=S \cup T
$$

for any finite subset $T$ of $X$.

Let $x_{1}$ be an arbitrary point in $X \backslash S$. To prove the theorem it is enough to show that

$$
L\left(S \cup\left\{x_{1}\right\}\right) \subseteq S \cup\left\{x_{1}\right\}
$$

Suppose there is a point $x_{0}$ in $L\left(S \cup\left\{x_{1}\right\}\right) \backslash\left(S \cup\left\{x_{1}\right\}\right)$. Then there is a function $g_{1} \in A$ satisfying the following properties: $g_{1}\left(x_{0}\right)=0,0 \dot{\notin} g_{1}(S)$ and $\left\|g_{1}\right\|<\frac{1}{2}$. Let 


$$
\delta=\inf _{x \in S}\left|g_{1}(x)\right|\left|1-g_{1}(x)\right|^{-1} .
$$

Clearly $0<\delta<1$. Now there is a function $g_{2}$ in $A$ such that $g_{2}\left(x_{0}\right)=0$, $g_{2}\left(x_{1}\right) \neq 0$ and $\left\|g_{2}\right\|<\frac{\delta}{2}$. Let $\lambda$ be any number in the closed bounded interval $\left[\frac{\delta}{2}, \delta\right]$ and let

$$
f(\lambda, x)=g_{1}(x)+\lambda g_{2}(x)-\lambda g_{1}(x) g_{2}(x) .
$$

If for some $x$ in $S f(\lambda, x)=0$, then

$$
\lambda=|\lambda|=\left|g_{2}(x)\right|^{-1}\left|g_{1}(x)\left\|1-\left.g_{1}(x)\right|^{-1} \geq\right\| g_{2} \|^{-1} \delta \geq \frac{2}{\delta} \cdot \delta=2 .\right.
$$

This contradicts the assumption that $\lambda \in\left[\frac{\delta}{2}, \delta\right]$, where $0<\delta<1$. Hence for any $\lambda$ in $\left[\frac{\delta}{2}, \delta\right]$ and any $x$ in $S f(\lambda, x) \neq 0$. Furthermore, it is not difficult to show that for all $\lambda$ in $\left[\frac{\delta}{2}, \delta\right]$ with one possible exception $\lambda_{0}$, $f\left(\lambda, x_{1}\right) \neq 0$. Hence, for any $x$ in $S \cup\left\{x_{1}\right\}$ and any $\lambda$ in $\left[\frac{\delta}{2}, \delta\right]$, where $\lambda \neq \lambda_{0}, f(\lambda, x) \neq 0$. Since $f\left(\lambda, x_{0}\right)=0, x_{0}$ does not belong to $L\left(S \cup\left\{x_{1}\right\}\right) \backslash$ $\left(S \cup\left\{x_{1}\right\}\right)$. This contradiction completes the proof of the theorem.

Direct verification yields the following

LEMMA 3.2. Let $S$ and $T$ be any two closed subsets of $X$, then

(i) $L(S) \subseteq L(T)$, whenever $S \subseteq T$, and

(ii) $L(S)=L(L(S))$.

We remark that in general $L$ is not a closure operator. This is demonstrated in the following examples.

EXAMPLE 3.3. Let $A$ be the algebra of all functions continuous on the closed unit bidisk,

$$
P=\{(z, w)|| z|\leq 1,| w \mid \leq 1\},
$$

in complex two-space which are analytic on the interior of the bidisk. It is known [8; p. 30] that if $f$ is in $A$ and $f$ vanishes at the origin, then $f$ is zero somewhere on the topological boundary of the bidisk. Now, if 


$$
S=\{(z, w)|| z|\leq 1,| w \mid=1\}
$$

and

$$
T=\{(z, w)|| z|=1,| w \mid \leq 1\},
$$

then $L(S \cup T) \neq L(S) \cup L(T)$.

EXAMPLE 3.4. Let $E$ be a totally disconnected perfect bounded set on the Riemann sphere $X$, such that the intersection of $E$ with any open set is either empty or has positive two dimensional Lebesgue measure. Let $A$ be the subalgebra of $C(X)$ consisting of all those $f$ in $C(X)$ which are analytic in $X \backslash E$. In [9; Theorem 1] Rudin showed that for all $f$ in $A, f(E)=f(X)$. Thus, $E$ is not an $L$-set.

It is easy to verify that an arbitrary intersection of $L$-sets is an $L$ set. Also, if $S$ is a hull-kernel closed set (for the terminology see Gamelin [4; p. 13]), then $L(S)=S$. Note that an $L$-set need not be a hull-kernel closed set.

We recall that a subset $S$ of $X$ is a set of antisymmetry (for $A$ ) if $f \in A$ and $f \mid S$ is real implies that $f \mid S$ is constant. As is well-known (Browder, [2; p. 137]) every set of antisymmetry is contained in a maximal set of antisymmetry. It is clear that a maximal set of antisymmetry is an $L$-set.

Finally, we remark that if $E$ is the essential set of $A$ in $X$, then $E$ is an $L$-set.

As noted above in general it is difficult to determine whether or not a closed subset $S$ of $X$ is an $L$-set. The following elementary result provides a simplification in the work involved.

LEMMA 3.5. Let $S$ be a closed subset of $X$.

(i) For any maximal ideal $M$

$$
L_{A}(S)=L_{M}(S)
$$

where

$$
L_{M}(S)=\bigcap_{f \in M} f^{-1} f(S)
$$

(ii) Suppose $X \backslash S$ contains at least two points and that for any pair of maximal ideals $M$ and $N$ in $X \backslash S$ 


$$
L_{A}(S)=L_{M \cap N}(S)
$$

Then either $S$ is an L-set or $L_{A}(S)=X$.

(iii) If $S$ is an $L$-set and $X \backslash S$ contains $n$ points, then there exist $n$ maximal ideals $M_{1}, \cdots, M_{n}$ such that

$$
L_{I}(S)=S,
$$

where

$$
I=\bigcap_{i=1}^{n} M_{i}
$$

In connection with part (ii) of Lemma 3.5 we remark that if the difference $X \backslash S$ is a singleton $\left\{x_{0}\right\}$, then $L(S)=X$ provided $x_{0}$ is not a peak point. For the definition of a peak point see Browder [2; p. 96].

We conclude this section with the following theorem.

THEOREM 3.6. Let $S$ be a closed subset of $X$ and let $x_{0} \in L(S) \backslash S$. Then $X$ contains a minimal closed subset $T$ such that $x_{0} \in L(T) \backslash T$.

We use Zorn's lemma in the proof. Let $T_{\alpha}$ be a chain of subsets of $X$ satisfying $x_{0} \in L\left(T_{\alpha}\right) \backslash T_{\alpha}$. Let $T=\bigcap_{\alpha} T_{\alpha}$. We claim that for each $f$ in $A$ there is a point $x_{f}$ in $T$ such that $f\left(x_{f}\right)=f\left(x_{0}\right)$. To see this, fix an $f$ in $A$ and choose $x_{\alpha}$ from $T_{\alpha}$ so that $f\left(x_{\alpha}\right)=f\left(x_{0}\right)$. Now let $x_{\beta}$ be a convergent subnet of $x_{\alpha}$ and let $x_{f}$ be its limit. Then $f\left(x_{f}\right)=$ $\lim f\left(x_{\beta}\right)=f\left(x_{0}\right)$ since $f$ is continuous. Thus $f\left(x_{0}\right) \in f\left(\cap T_{\alpha}\right)$ for each $f$.

4. Separating Algebras. We will say that an algebra $A$ on $X$ is a separating algebra if every closed set in $X$ is an $L$-set. Clearly, if $A$ is a separating algebra on $X$ and $B$ is an algebra on $X$ containing $A$, then $B$ is also a separating algebra. In view of the results of section 3 we note that if $A$ is a separating algebra then $L$ is a closure operator and $L$ defines a topology on $X$ which is equivalent to the given topology. This is the content of the following characterization of separating algebras.

THEOREM 4.1. If $A$ is an algebra on $X$, then the following assertions are equivalent.

(1) $A$ is a separating algebra.

(2) For each pair of closed sets $S_{1}$ and $S_{2}$ in $X$ such that $L\left(S_{i}\right)=S_{i}$, $i=1,2$,

$$
L\left(S_{1} \cup S_{2}\right)=L\left(S_{1}\right) \cup L\left(S_{2}\right) .
$$


(3) For each closed set $S$ and a point $x \notin S$ there is a function $f$ in $\operatorname{Ker} x$,

$$
\operatorname{Ker} x=\{f \in A \mid f(x)=0\},
$$

such that

$$
Z(f) \cap S=\phi
$$

where

$$
Z(f)=\{x \in X \mid f(x)=0\} .
$$

We will only show that (2) implies (1). If statement (2) is satisfied then the $L$-sets are the closed sets of a topology $\tau$. Now the relations

$$
f^{-1}(C) \subset L\left(f^{-1}(C)\right)=\bigcap_{g \in A} g^{-1} g\left(f^{-1}(C)\right) \subset f^{-1} f f^{-1}(C)=f^{-1}(C)
$$

show that $f^{-1}(C)$ is a $\tau$-closed set for each closed set $C$ and each $f$ in $A$. Thus each $f$ in $A$ is $\tau$ continuous. Since $A$ separates points of $X, \tau$ is a Hausdorff topology. But since $\tau$ is contained in the original (compact) topology it is equivalent to it.

We recall that $A$ is a Dirichlet algebra on $X$ if the real parts of the functions in $A$ are uniformly dense in the real continuous functions on $X$. The prototype of a Dirichlet algebra is the algebra of continuous functions on the unit circle whose Fourier coefficients vanish on the negative integers.

THEOREM 4.2. Every Dirichlet algebra is a separating algebra.

Let $S$ be a closed subset of $X$ and $x_{0}$ not in $S$. Choose a real-valued continuous function $f$ satisfying $f\left(x_{0}\right)=0$ and

$$
\inf _{x \in S} f(x)=1 \text {. }
$$

Now let $g=u+i v$ be a function in $A$ satisfying $\|f-u\|<1 / 2$. Then the inequalities

$$
u\left(x_{0}\right)<\frac{1}{2}<\inf _{x \in S} u(x)
$$

show that $g\left(x_{0}\right)$ is not in $g(S)$. This completes the proof of the theorem.

We remark that the example of the disk algebra shows that a separating algebra need not be a Dirichlet algebra. 
Before proving our next result we recall some well-known facts (Hoffman and Singer [7; p. 218]) about maximal algebras. Suppose $A$ is a maximal subalgebra of $C(X)$ and suppose $S$ is a closed subset of $X$. Let $A_{0}=\left\{f \in C(X)|f| S \in A_{S}\right\}$, then $A_{0}$ is closed and $A \subseteq A_{0} \subseteq C(X)$. Thus either $A_{S}=C(S)$ or every function $f$ in $C(X)$ such that $f(S)=0$ is in A.

THEOREM 4.3. If $A$ is a maximal essential subalgebra of $C(X)$, then $A$ is a separating algebra.

Let $S$ be a closed set and let $x_{0}$ be a point not in $S$. If $x_{0}$ is in $L(S)$, then $S \cup\left\{x_{0}\right\}=X$. To see this let $f$ be a function in $C(X)$ whose restriction to $S \cup\left\{x_{0}\right\}$ is zero. If $f$ is not in $A$, then the algebra $(A, f)$ generated by $A$ and $f$ is dense in $C(X)$. Thus each $g$ in $C(X)$ satisfying $g\left(x_{0}\right)=0$ and $g(x)=1$ for each $x$ in $S$ can be approximated by polynomials in $f$. That is

$$
\left\|\sum_{i=1}^{n} a_{i} f^{i}-g\right\|<\frac{1}{4} \quad \text { for some } a_{i} \in A .
$$

Since $f\left(S \cup\left\{x_{0}\right\}\right)=\{0\}$ we have

$$
\left|a_{0}(x)-g(x)\right|<\frac{1}{4} \quad \text { for all } x \text { in } S \cup\left\{x_{0}\right\}
$$

This shows that $a_{0}\left(x_{0}\right) \notin a_{0}(S)$; whence $x_{0} \notin L(S)$. This contradiction shows that every continuous function vanishing on $S \cup\left\{x_{0}\right\}$ belongs to $A$. Since $A$ is essential, $X$ has no proper closed subset $T$ such that each continuous function vanishing on $T$ belongs to $A$. Now it is well-known [6; p. 304] that the Silov boundary of an algebra $A$ maximal in $C(X)$ is $X$ itself. This means that $x_{0}$ is an isolated point of the Silov boundary $X$. Thus $x_{0}$ is a peak point for $A$ and $x_{0} \notin L(S)$.

The results of Bear [1] show that the study of function algebras can effectively be reduced to the study of essential algebras. Thus it is not surprising that we can weaken the hypothesis of Theorem 4.3 to obtain

THEOREM 4.4. If $A$ is a maximal subalgebra of $C(X)$, then $A$ is a separating algebra.

We have seen that if $S$ is a closed set and $x_{0}$ is in $L(S) \backslash S$, then $S \cup\left\{x_{0}\right\}$ contains the essential set $E$ of the algebra $A$. Thus $E \subseteq S \cup\left\{x_{0}\right\}$. 
By Theorem 4.3 we may assume that $A$ is not essential. Thus $E$ is a proper subset of $X$ and

$$
A=\left\{f \in C(X)|f| E \in A_{E}\right\} .
$$

Suppose $x_{0}$ is not in $E$. Let $g$ be any function in $C(X)$ such that $g\left(x_{0}\right)$ $\notin g(S)$ and $g|E=f| E$ for some $f$ in $A$. Then $g \in A$ and we conclude that $x_{0} \notin L(S)$. On the other hand, if $x_{0}$ is in $E$ then $x_{0}$ is an isolated point in $E$ since $E \backslash\left\{x_{0}\right\}$ is contained in $S$. Since $A$ is maximal, there is a function $f$ in $A$ such that $f\left(x_{0}\right)=\|f\|=1$ and $|f(x)| \leq c<1$ on $E \backslash\left\{x_{0}\right\}$. Extend $f \mid E \backslash\left\{x_{0}\right\}$ to a continuous function $F$ defined on $S$ without increasing the range. Now extend $F$ continuously to all of $X$. This extension of $F$ belongs to $A$ because its restriction to $E$ belongs to $A_{E}$. Also the extended function separates $x_{0}$ from $S$.

In [7; p. 221] Hoffman and Singer give an example of a function algebra which is pervasive but not maximal. The proof of the next theorem is similar to the one given above.

THEOREM 4.5 If $A$ is a pervasive subalgebra of $C(X)$, then $A$ is a separating algebra.

We pause for a moment to discuss the scope of the foreign result. In the above proofs we used the fact that for function algebras which are maximal or pervasive the Silov boundary, $\Gamma$, is the whole space $X$. Thus it is natural to inquire whether the condition $\Gamma=X$ is sufficient to guarantee that all closed sets are $L$-sets. The following example shows that in general the condition $X=\Gamma$ is not sufficient.

EXAmple 4.6. As before let $P=\{(z \cdot w)|| z|\leq 1| w \mid, \leq 1\}$. Let $T=$ $\{(z, w)|| z \mid=1$ or $|w|=1\}$ denote the topological boundary and let $A$ be the bidisk algebra on $P$. Let $\theta=(0,0)$ and define $A_{0}$ by

$$
A_{0}=\left\{f \in C(P)|f| T \cup\{\theta\} \in A_{T \cup\{\theta\}}\right\}
$$

Then $A_{0}$ is not a separating algebra on the connected space $P$ and the Silov boundary $A_{0}$ is all of $P$ since each point in the interior of $P \backslash\{\theta\}$ is a peak point of $A_{0}$.

We conclude this paper with the following important unanswered questions. 
(1) Is there a non-separating essential algebra $A$ on a (connected) space $X$ for which $M_{A}=\Gamma_{A}=X$ ?

(2). Let $H_{\alpha}^{\infty}$ denote the function algebra obtained by restricting $H^{\infty}$ to the fiber $M_{\alpha}$. (For the terminology used here we refer the reader to Hoffman [5; p. 187]). Is every closed subset of the maximal ideal space, $M_{\alpha}$, of $H_{\alpha}^{\infty}$ an $L$-set? It would be interesting to see a proof of this using Carleson's Corona Theorem.

\section{BIBLIOGRAPHY}

[1] Bear, H. S., Complex function algebras, Trans Amer. Math. Soc., 90 (1959), 383-393.

[ 2 ] Browder, A., Introduction to Function Algebras, W. A. Benjamin, N. Y., 1969.

[ 3 ] Carleson, L., Interpolation by bounded analytic functions and the corona problem, Ann. of Math., 76 (1962), 547-559.

[ 4 ] Gamelin, T. W., Uniform Algebras, Prentice-Hall, Englewood Cliffs, N. J., 1969.

[5] Hoffman, K., Banach Spaces of Analytic Functions, Prentice-Hall, Englewood Cliffs, N. J., 1962.

[6] Hoffman, K. and Singer, I. M., Maximal subalgebras of $C(\Gamma)$, Amer. J. Math., 79 (1957), 295-305.

[ 7 ] Hoffman, K. and Singer, I. M., Maximal algebras of continuous functions, Acta Math., 103 (1960), 217-241.

[8] Hörmander, L., An Introduction to Complex Analysis in Several Variables, Van Nostrand, Princeton, N. J., 1966.

[ 9 ] Rudin, W., Subalgebras of spaces of continuous functions, Proc. Amer. Math. Soc., 7 (1956), 825-830.

[10] Weiss, M. L., Some separation properties in sup-norm algebras of continuous functions, Function Algebras, Scott, Foresman and Co., 1966, 93-97.

[11] Wilken, D. R., Approximate normality and function algebras on the interval and the circle, Function Algebras, Scott, Foresman and Co., 1966, 98-111.

University of Hawaii 